Pacific Journal of Mathematics

ON THE MEIJER TRANSFORM OF GENERALIZED

R. S. PATHAK 


\title{
ON THE MEIJER TRANSFORM OF GENERALIZED FUNCTIONS
}

\author{
R. S. PATHAK
}

An extension of the Meijer transform to a certain space generalized functions (distributions) is provided. The validity of the inversion formula in the distributional sense is established. Characterization theorem for the distributional Meijer transform is proved and a structure formula for the Meijer transformable generalized functions is given. An operation-transform formula is obtained, which together with the inversion formula, is applied in solving certain integrodifferential equations.

1. Introduction. During the past decade a number of integral transforms have been extended to various classes of generalized functions. Some of these extensions have been incorporated by Zemanian in his monograph [14]. The Meijer transform of ordinary functions has been studied by many authors [2], [5], [8], and [9] but its distributional theory has not yet been explored. The aim of the present paper is to extend the Meijer transform to a certain space of generalized functions and to establish certain related results. The novelity of the extension lies in the construction of the testing function space where instead of taking a differential operator one has to think of an integrodifferential operator of a certain kind.

Let $k, m$, and $z$ be complex variables, let $t, \sigma$, and $\omega$ be real variables in $R^{1}$, and set $s=\sigma+i \omega$. The Whittaker functions $W_{k, m}(z)$ and $M_{k, m}(z)$ are defined by the series [7, pp. 9-10]

$$
M_{k, m}(z)=z^{(1 / 2)+m} e^{-(1 / 2) z}{ }_{1} F_{1}\left(\frac{1}{2}+m-k ; 1+2 m ; z\right)
$$

and

(2) $W_{k, m}(z)$

$$
=\frac{\pi}{2 \sin m \pi}\left(\frac{-M_{k, m}(z)}{\Gamma\left(\frac{1}{2}-m-k\right) \Gamma(1+2 m)}+\frac{M_{k,-m}(z)}{\Gamma\left(\frac{1}{2}+m-k\right) \Gamma(1-2 m)}\right) .
$$

The function $M_{k, m}(z)$ is analytic everywhere except at the points $2 m=-1,-3,-5, \cdots$, where it has simple poles. At these points, however, the function $M_{k, m}(z) / \Gamma(1+2 m)$ is analytic. The function $W_{k, m}(z)$ is defined for all real and complex values of $k, m$, and $z$. It is a many valued function of $z$. We shall take as its principal branch that which lies in the $z$-plane cut along the negative real 
axis. It is a fact that $W_{k, m}(z)=W_{k,-m}(z)[7, \mathrm{p} .11]$, therefore, we lose no generality in restricting according to $0 \leqq \operatorname{Re} m<\infty$.

The asymptotic behaviors of Whittaker functions for large values of $z$ are the following [2, pp. 734-735]. For any fixed $\varepsilon>0$ and $|z| \rightarrow \infty$,

$$
\begin{aligned}
& e^{-1 / 2 z} W_{k, m}(z)= e^{-1 / 2 z} z^{k}\left\{1+0\left(|z|^{-1}\right)\right\}\left(-\frac{3}{2} \pi+\varepsilon<\arg z<\frac{3}{2} \pi-\varepsilon\right) \\
& e^{1 / 2 z} M_{k, m}(z)= \frac{\Gamma(1+2 m)}{\Gamma\left(\frac{1}{2}-k+m\right)} e^{z} z^{-k}\left\{1+O\left(|z|^{-1}\right)\right\} \\
&+\frac{\Gamma(1+2 m)}{\Gamma\left(\frac{1}{2}+k+m\right)} e^{-(k-m-1 / 2) \pi i} z^{k}\left\{1+O\left(|z|^{-1}\right)\right\} \\
& e^{1 / 2 z} M_{k, m}(z)= \frac{\Gamma(1+2 m)}{\Gamma\left(\frac{1}{2}-k+m\right)} z^{-k}\left\{1+O\left(|z|^{-1}\right)\right\} \\
&+\frac{\Gamma(1+2 m)}{\Gamma\left(\frac{1}{2}+k+m\right)} e^{(k-m-1 / 2) \pi i} z^{k}\left\{1+O\left(|z|^{-1}\right)\right\} \\
&\left(-\frac{3}{2} \pi+\varepsilon<\arg z<\frac{1}{2} \pi-\varepsilon\right) .
\end{aligned}
$$

The other results that we shall need are the following differentiation formula $[7$, p. 25]

$$
\frac{d}{d x}\left\{e^{-1 / 2 x} x^{m-1 / 2} W_{k, m}(x)\right\}=-e^{-1 / 2 x} x^{m-1} W_{k+1 / 2, m-1 / 2}(x)
$$

and the indefinite integral $[2, \mathrm{p} .733]$

$$
\begin{aligned}
(x-t) & \int e^{1 / 2 x s} M_{k-1 / 2, m}(x s) e^{-1 / 2 t s} W_{k+1 / 2, m}(t s) s^{-1} d s \\
= & \frac{1}{m-k}\left[2 m x^{1 / 2} s^{-1 / 2} e^{1 / 2 x s} M_{k, m-1 / 2}(x s) e^{-1 / 2 t s} W_{k+1 / 2, m}(t s)\right. \\
& \left.\quad-(k+m) t^{1 / 2} s^{-1 / 2} e^{1 / 2 x s} M_{k+1 / 2, m}(x s) e^{-1 / 2 t s} W_{k, m-1 / 2}(t s)\right] .
\end{aligned}
$$

Now, we reproduce Meijer's inversion theorem in the original form.

THEOREM (Meijer). Let $F(s)$ be an analytic function on the half plane $\operatorname{Re} s>a \geqq 0$. For some real constant $c>a$, let the integral 


$$
\int_{-\infty}^{\infty}|F(c+i y)| d y
$$

converge. Moreover, assume that $F(s)$ is bounded according to $|F(s)|<A, A>0$ for $\operatorname{Re} s \geqq c$ and that $F(x+i y) \rightarrow 0$ as $x \rightarrow \infty$ uniformly for $-\infty<y<\infty$. Finally, assume that $\operatorname{Re} k \leqq-\operatorname{Re}<1 / 2$. Then, for $\operatorname{Re} s>c$,

$$
F(s)=\int_{0}^{\infty} e^{-1 / 2 s t} W_{k+1 / 2, m}(s t)(s t)^{-k-1 / 2} f(t) d t
$$

where

$$
f(t)=\frac{\Gamma(1-k+m)}{2 \pi i \Gamma(1+2 m)} \int_{c-i \infty}^{c+i \infty} e^{1 / 2 t z} M_{k-1 / 2, m}(t z)(t z)^{k-1 / 2} F(z) d z .
$$

2. An integrodifferential operator. From the differential equation satisfied by Whittaker functions [7] it is a simple exercise to show that the kernels

$$
K(x) \triangleq e^{-1 / 2 x} W_{k+1 / 2, m}(x) x^{-k-1 / 2}
$$

and

$$
H(x) \triangleq \frac{\Gamma(1-k+m)}{\Gamma(1+2 m)} e^{1 / 2 x} M_{k-1 / 2, m}(x) x^{k-1 / 2}
$$

satisfy the integrodifferential equations

$$
\Delta_{x} K(\alpha x)=-\alpha K(\alpha x)
$$

and

$$
\nabla_{x} H(\alpha x)=\alpha H(\alpha x)
$$

respectively, where $\Delta_{x}$ and $\nabla_{x}$ are defined as below:

$$
\begin{aligned}
& \Delta_{x} \triangleq \Delta_{x}^{k, m} \triangleq x^{-1}\left(x^{-2 k} D^{-1} x^{2 k-1}\right)\left(x^{1-k+m} D x^{k-m}\right)\left(x^{1-k-m} D x^{k+m}\right) \\
& \nabla_{x} \triangleq \nabla_{x}^{k, m} \triangleq x^{-1}\left(x^{2 k} D^{-1} x^{-2 k-1}\right)\left(x^{1+k+m} D x^{-k-m}\right)\left(x^{1+k-m} D x^{-k+m}\right)
\end{aligned}
$$

in $\Delta_{x}$ we interpret $D^{-1}=\int_{\infty}^{x} \cdots d t$ and in $\nabla_{x}, D^{-1}=\int_{0}^{x} \cdots d t$.

REMARK. The operator $\Delta_{x}$ can be applied on any $C^{\infty}(R+)$ function $\phi$ any number of times which satisfies the asymptotic orders

$$
\phi^{(r)}(x)=O\left(x^{\alpha-r}\right), \quad x \longrightarrow \infty, \quad r=0,1,2, \cdots
$$

where $\alpha+2 \operatorname{Re} k<0$. If $\phi^{(r)}(x)$ possess exponentially small aymptotic orders as $x \rightarrow \infty$, then this condition does not apply. The operator $\nabla_{x}$ can be applied to any $C^{\infty}(R+)$ function $\phi$ any number of times which satisfies the asymptotic orders 


$$
\phi^{(r)}(x)=O\left(x^{\alpha-r}\right), \quad x \longrightarrow 0+, \quad r=0,1,2, \cdots
$$

where $\alpha>2$ Re $k$. Furthermore, if $\phi(x) \in C^{\infty}(R+)$ is of compact support in $(0, \infty)$ then the two interpretations of $D^{-1}$ are identical and the aforesaid asymptotic order conditions are not required.

Some properties of these operators are described below.

Lemma 1. Let $\phi \in C^{\infty}(R+)$ with the asymptotic orders (16) (or (17) in case of $\left.\nabla_{x}\right)$, then the integration operator $\left(x^{-2 k} D^{-1} x^{2 k-1}\right)$ and the differentiation operator $\left(x^{1-k+m} D x^{k-m}\right)$ occuring in $\Delta_{x}$ (or in $\nabla_{x}$ ) when acting on $\phi$ in succession are commutative.

Proof. A simple computation shows that

$$
\begin{aligned}
& \left(x^{1-l+m} D x^{l k-m}\right)\left(x^{-2 k} D^{-1} x^{2 k-1}\right) \dot{\phi}(x) \\
& =x^{1-k+m} D x^{-m-k} \int_{\infty}^{x} y^{2 k-1} \phi(y) d y \\
& =\dot{\phi}(x)-(m+k) x^{-2 k} \int_{\infty}^{x} y^{2 k-1} \phi(y) d y, \quad \alpha+\operatorname{Re} 2 k<0
\end{aligned}
$$

and

$$
\begin{aligned}
& \left(x^{-2 k} D^{-1} x^{2 k-1}\right)\left(x^{1-k+m} D x^{k-m}\right) \dot{\phi}(x) \\
& \quad=x^{-2 k} D^{-1} x^{k+m}\left[x^{k-m} \phi^{\prime}(x)+(k-m) x^{k-m-1} \dot{\phi}(x)\right] \\
& \quad=\dot{\phi}(x)-(m+k) x^{-2 k} \int_{\infty}^{x} y^{2 k-1} \dot{\varphi}(y) d y, \quad \alpha+\operatorname{Re} 2 k<0 .
\end{aligned}
$$

This proves the lemma.

COROLlaRY. The differentiation and integration operators as defined in Lemma 1 occuring in $\Delta_{x}$ and $\nabla_{x}$ when acting on $\phi \in C^{\infty}(R+)$ satisfying (16) in case of $\nabla_{x}$ and (17) in case of $\nabla_{x}$ can be switched in any order.

Proof. Since two differentiation operators are commutative the result follows in view of Lemma 1 .

3. The testing function space $\mathscr{I}_{a}^{k, m}(I)$. Let $I$ denote the open interval $(0, \infty), x \in I$ and let $a$ be a real positive number and $k$ and $m$ be complex numbers. Assume that $\operatorname{Re} m \geqq 0$. Now, define $\mathscr{F}_{a}^{k, m}(I)$ to be the collection of all infinitely differentiable complex valued functions $\phi(x)$ on $I$ with the properties (16) and

$$
\rho_{n}(\dot{\phi}) \triangleq \rho_{a, n}^{k, m}(\dot{\phi}) \triangleq \sup _{0<x<\infty}\left|e^{a x} x^{k+m} \Delta_{x}^{n} \dot{\phi}(x)\right|<\infty, \quad n=0,1,2, \cdots
$$

where $\Delta_{x}$ is the integrodifferential operator defined by (14). The sequence $\left\{\rho_{n}\right\}_{n=0}^{\infty}$ is a separating collection of seminorms $[14, \mathrm{p} .8]$ 
which generates the topology of $\mathscr{I}_{a}^{k, m}(I)$. It can be readily seen that $\mathscr{I}_{a}^{k, m}(I)$ is a locally convex, sequentially complete, Hausdorff topological vector space. The dual space of $\mathscr{f}_{a}^{k, m}(I)$ is denoted by $\mathscr{I}_{a}^{k, m^{\prime}}(I)$.

Let $D(I)$ denote the space of infinitely differentiable complex valued functions with compact support on $I$, equipped with the usual topology. The dual space $D^{\prime}(I)$ is the space of Schwartz distributions on $I\left[14\right.$, pp. 33-34]. It is easily seen that $D(I) \subset \mathscr{J}_{a}^{k, m}(I)$ and that the topology of $D(I)$ is stronger than that induced on it by $\mathscr{J}_{a}^{k, m}(I)$. Hence the restriction of any $f \in \mathscr{F}_{a}^{k, m^{\prime}}(I)$ to $D(I)$ is in $D^{\prime}(I)$.

For $0<a<b$ the space $\mathscr{I}_{b}^{k, m} \subset \mathscr{I}_{a}^{k, m}$, and the topology of $\mathscr{I}_{b}^{k, m}$ is stronger than the topology induced on it by $\mathscr{J}_{a}^{k, m}$. Consequently, the restriction of $f \in \mathscr{J}_{a}^{k, m^{\prime}}$ to $\mathscr{J}_{b}^{k, m}$ is in $\mathscr{I}_{b}^{k, m^{\prime}}$ and the convergence in $\mathscr{J}_{a}^{k, m^{\prime}}$ implies convergence in $\mathscr{F}_{b}^{k, m^{\prime}}$.

We notice that for every fixed $s$ such that $\operatorname{Re} s>a>0$ and $\operatorname{Re} m \geqq 0,(s t)^{-k-1 / 2} e^{-1 / 2 s t} W_{k+1 / 2, m}(s t)$ is a member of $\mathscr{J}_{a}^{k, m}(I)$.

4. The Meijer transform of generalized functions. Let $f$ be a member of $\mathscr{J}_{a}^{k, m^{\prime}}$ for some $k, m$, and $a$. Then, from the preceding argument it is clear that there exists some real number $\sigma_{f} \geqq 0$, depending upon $f$ such that $f \in \mathscr{I}_{a}^{k, m^{\prime}}$ for all $a>\sigma_{f}$ and $f \notin \mathscr{I}_{a}^{k, m}$ for every $a<\sigma_{f}$.

Now recall the definition (10) of $K(z)$. Since $K(s t) \in \mathscr{I}_{a}^{k, m}$ for every $s$ such that $\operatorname{Re} s>a$ and $\operatorname{Re} m \geqq 0$, we may define the distributional Meijer transform of $f$ by

$$
F(s) \triangleq \mathscr{M}_{k, m} f(s) \triangleq\langle f(t), K(s t)\rangle, \quad \operatorname{Re} s>\sigma_{f}
$$

where $\sigma_{f}$ is called the abscissa of definition.

LEMMA 2. Let $\operatorname{Re} m \geqq 0$, and let $a$ and $b(>a)$ be two real numbers. Then, for $\operatorname{Re} \zeta \geqq b, \zeta \neq 0,-\pi<\arg \zeta \leqq \pi$ and $0<t<\infty$,

$$
\left|e^{a t}(\zeta t)^{m-1 / 2} e^{-1 / 2 \zeta t} W_{k+1 / 2, m}(\zeta t)\right|<A\left(1+|\zeta|^{2 r}\right)
$$

where $A$ is a constant independent of $\zeta$ and $t$, and $\lambda_{r}=\operatorname{Re}(m+k)$.

Proof. The proof can be given by following the technique of Zemanian [14, p. 184] and using the estimates

$$
\left|z^{m-1 / 2} e^{-1 / 2 z} W_{k+1 / 2, m}(z)\right|<A \text { for } \operatorname{Re} m \geqq 0 \text { and }|z| \leqq 1
$$

and

$$
\left|z^{m-1 / 2} e^{-1 / 2 z} W_{k+1 / 2, m}(z)\right|<B|z|^{\lambda_{r}} e^{-\operatorname{Re} z} \text { for }|z|>1
$$


These estimates can easily be obtained from the series representation (2) and the asymptotic expansion (3).

THEOREM 1. (Analiticity of $F(s)$ ). For Re $s>\sigma_{f}$, let $F(s)$ be the Meijer transform of $f \in \mathscr{F}_{a}^{k, m^{\prime}}$ defined by (19). Then, $F(s)$ in analytic and

$$
\frac{d}{d s} F(s)=\left\langle f(t), \frac{\partial}{\partial s} K(s t)\right\rangle
$$

where $\operatorname{Re} m \geqq 0$.

Proof. Using the differentiation formula (6), series representation (2) and the asymptotic expansion (3) we observe that $\partial / \partial s K(s t) \epsilon$ $\mathscr{F}_{a}^{k, m}(I)$ and hence the right-hand side of (21) is meaningful. Using Lemma 2 and following the technique of Zemanian [14] used in proving Theorem $6.5-1$, p. 185, the proof can be given.

5. Inversion and uniqueness. In this section we shall prove an inversion theorem for the distributional Meijer transform and then deduce an uniqueness theorem.

Lemma 3. For $\operatorname{Re} s>\sigma_{f}$, let $F(s)$ be defined by (19). Let $\phi \in D(I)$, and set

$$
\psi(s)=\int_{0}^{\infty} K(s t) \phi(t) d t, \quad \operatorname{Re} s>0
$$

Then, for any fixed real number $r$ in $(0, \infty)$,

$$
\int_{-r}^{r} \psi(s)\langle f(\tau), K(s \tau)\rangle d \omega=\left\langle f(\tau), \int_{-r}^{r} \psi(s) K(s t) d \omega\right\rangle
$$

where $s=\sigma+i \omega$ and $\sigma$ is fixed with $\sigma>\max \left(0, \sigma_{f}\right)$.

Proof. Consider the integral

$$
I(\tau)=\int_{-r}^{r} \psi(s) K(s \tau) d w
$$

where $\max \left(0, \sigma_{f}\right)<a<\sigma$. For $\operatorname{Re} m \geqq 0$ we can apply the operator $\Delta_{\tau}$ within the integral sign in (23) and write

$$
\begin{aligned}
\left|e^{a \tau} \tau^{k+m} \Delta_{\tau}^{(n)} I(\tau)\right|= & \left|\int_{-r}^{r} \psi(s) e^{a \tau} s^{n} K(s t) d \omega\right| \\
\leqq & \int_{-r}^{r}\left|\psi(s) s^{n-k-m}\right| A\left(1+|s|^{\lambda}\right) d \omega<\infty \\
& (\text { by Lemma 2). }
\end{aligned}
$$


This proves that $I(\tau) \in \mathscr{J}_{a}^{k, m}$ and hence the right-hand side of (22) is meaningful. The equality (22) can be proved by following the technique of Riemann sums [14, pp. 187-188].

LEMMA 4. Let $\phi(x) \in D(I)$ and let its support be contained in $[c, d]$, where $0<c<d<\infty$. Let $\operatorname{Re} m \geqq 0, \operatorname{Re}(m-k) \geqq 0$ and Re $k<1 / 2$. Then for fixed $\sigma>a \geqq 0$,

$$
W_{r}(\tau) \triangleq \frac{1}{2 \pi} \int_{-r}^{r} K(s \tau) \int_{0}^{\infty} \phi(t) H(s t) d t d \omega, \quad s=\sigma+i \omega
$$

converges in $\mathscr{J}_{a}^{k, m}$ to $\phi(\tau)$ as $r \rightarrow \infty$.

Proof. In view of the definitions of the operators $\Delta_{x}$ and $\nabla_{x}$, we have

$$
\begin{aligned}
\Delta_{\tau}^{(n)} W_{r}(\tau) & =\frac{1}{2 \pi} \int_{-r}^{r} \Delta_{\tau}^{(n)} K(s \tau) \int_{0}^{\infty} \phi(t) H(s t) d t d \omega \\
& =\frac{1}{2 \pi} \int_{-r}^{r} K(s \tau) \int_{0}^{\infty} \phi(t)(-1)^{n} \nabla_{t}^{(n)} H(s t) d t d \omega \\
& =\frac{1}{2 \pi} \int_{-r}^{r} K(s \tau) \int_{0}^{\infty} \phi_{n}(t) H(s t) d t d \omega
\end{aligned}
$$

where $\phi_{n}(t) \triangleq \Delta_{t}^{(n)} \phi(t)$, on integrating by parts with respect to $t n$ times. Changing the order of integration we can write

$$
\Delta_{\tau}^{(n)} W_{r}(\tau)=\int_{c}^{d} U_{r}(t, \tau) \phi_{n}(t) d t
$$

where

$$
\begin{aligned}
U_{r}(t, \tau)= & \frac{1}{2 \pi i} \frac{\Gamma(m-k)}{\Gamma(2 m+1)} \frac{\tau^{-k-1 / 2}}{t-\tau} t^{-k-1 / 2} \\
& \times\left[2 m t^{1 / 2} s^{-1 / 2} e^{1 / 2 s t} M_{k, m-1 / 2}(s t) e^{-1 / 2 s \tau} W_{k+1 / 2, m}(s \tau)\right. \\
& \left.-(k+m) \tau^{1 / 2} s^{-1 / 2} e^{1 / 2 s t} M_{k+1 / 2, m}(s t) e^{-1 / 2 s \tau} W_{k, m-1 / 2}(s \tau)\right]_{\sigma-i r}^{\sigma+i r} .
\end{aligned}
$$

Now, break up the integration (24) into integrations on $c<t<$ $\tau-\delta, \quad \tau-\delta<t<\tau+\delta$ and $\tau+\delta<t<d$ where $0<\delta<c$ and denote the corresponding integrals by $I_{1}, I_{2}$, and $I_{3}$ respectively. We shall show first that

$$
V_{r}(\tau) \triangleq e^{a \tau} \tau^{k+m}\left[I_{2}(\tau)-\phi_{n}(\tau)\right], \quad(n=1,2, \cdots)
$$

converges uniformly to zero on $0<\tau<\infty$ as $r \rightarrow \infty$. If either $\tau+\delta \leqq c$ or $\tau-\delta \geqq d$, then $I_{2} \equiv 0$ and $\phi_{n}(\tau) \equiv 0$. Therefore, we consider the case $c-\delta<\tau<d+\delta$.

Now, for $s=\sigma \pm i r$ where $\sigma>0$ is fixed, using the asymptotic orders (3), (4), and (5) we can write 


$$
\begin{aligned}
V_{r}(\tau)= & e^{a \tau} \frac{\tau^{m+k}}{\pi}\left[\int_{\tau-\delta}^{\tau+\delta} \frac{\sin r(t-\tau)}{t-\tau} e^{\sigma(t-\tau)} \phi_{n}(t) d t\right. \\
& +\int_{\tau-\delta}^{\tau+\delta} \frac{\sin r(t-\tau)}{t-\tau} e^{\sigma(t-\tau)} \phi_{n}(t)\left\{O\left(\frac{1}{|s t|}\right)+O\left(\frac{1}{|s \tau|}\right)\right. \\
& \left.+O\left(\frac{1}{|s t|}\right) O\left(\frac{1}{|s \tau|}\right)\right\} d t \\
& -(k+m)\left\{1+O\left(\frac{1}{|s \tau|}\right)\right\} \int_{\tau-\delta}^{\tau+\delta} e^{\sigma(s-\tau)} t^{-1}\left(\frac{e^{i r}(t-\tau)}{\sigma+i r}-\frac{e^{-i r}(t-\tau)}{\sigma-i r}\right) \\
& \left.\times \phi_{n}(t)\left\{1+O\left(\frac{1}{|s t|}\right)\right\} d t\right] \\
& -e^{a \tau} \tau^{k+m} \phi_{n}(\tau) .
\end{aligned}
$$

It is a simple exercise to show that the second and third terms on the right-hand side of (26) are uniformly bounded on the domain

$$
\Omega_{1} \triangleq\{(t, \tau): c<t<d, c-\delta<\tau<d+\delta\}
$$

by $\varepsilon / 3$ for all $r>1$ and $\delta$ sufficiently small, say $\delta=\delta_{1}$.

Next, the difference of the first and last term in (26) can be written as

$$
\frac{1}{\pi} \int_{-\delta}^{\delta} G(x, \tau) \sin (r x) d x+e^{a \tau} \tau^{k+m} \phi_{n}(\tau)\left[\frac{1}{\pi} \int_{-\delta r}^{\delta r} \frac{\sin y}{y} d y-1\right]
$$

where $G(x, \tau)$ is defined by

$$
\begin{aligned}
G(x, \tau) & =e^{a \tau} \tau^{m+k} \frac{1}{x}\left[e^{\sigma x} \phi_{n}(\tau+x)-\phi_{n}(\tau)\right] & & x \neq 0 \\
& =e^{a \tau} \tau^{m+k} \phi_{n}^{\prime}(\tau) & & x=0 .
\end{aligned}
$$

Then $G(x, \tau)$ is a continuous function of $(x, \tau)$ for $x+\tau>0$ and $\tau>0$. Consequently, the first term in (27) can be made less than $\varepsilon / 3$ for all $r>1$ by choosing $\delta$ small enough, say $\delta=\delta_{2}$. Now, fix $\delta=\min \left(\delta_{1}, \delta_{2}\right)$. Since the second term in (27) converges uniformly to zero on $0<\tau<\infty$ as $r \rightarrow \infty$, we conclude that

$$
\varlimsup_{r \rightarrow \infty}\left|V_{r}(\tau)\right| \leqq \varepsilon \text {. }
$$

Since $\varepsilon>0$ is arbitrary, $V_{r}(\tau)$ converges uniformly to zero on $0<\tau<\infty$ as $r \rightarrow \infty$.

Following the technique of Zemanian [14, pp. 191-194] it can be shown that

$$
e^{a \tau} \tau^{k+m} I_{1}(\tau) \text { and } \quad e^{a \tau} \tau^{k+m} I_{3}(\tau)
$$

converge uniformly to zero on $0<\tau<\infty$ as $r \rightarrow \infty$. This proves the lemma. 
Now, we are able to establish the following inversion theorem.

THEOREM 2 (Inversion). Let $F(s)$ be the distributional Meijer transform of $f \in \mathscr{J}_{a}^{k, m}(I)$ for $\operatorname{Re} s>\sigma_{f}$ defined by

$$
F(s) \triangleq\left\langle f(t),(s t)^{-k-1 / 2} e^{-1 / 2 s t} W_{k+1 / 2, m}(s t)\right\rangle,
$$

where $\operatorname{Re} m \geqq 0, \operatorname{Re}(m-k) \geqq 0$ and $\operatorname{Re} k<1 / 2$. Then for each $\phi(x) \in D(I)$,

$$
\begin{aligned}
\lim _{r \rightarrow \infty} & \left\langle\frac{1}{2 \pi i} \frac{\Gamma(1+m-k)}{\Gamma(1+2 m)} \int_{\sigma-i r}^{o+i r} F(s)(s t)^{-k-1 / 2} e^{1 / 2 s t} M_{k-1 / 2, m}(s t) d s, \phi(t)\right\rangle \\
& =\langle f(t), \phi(t)\rangle
\end{aligned}
$$

where $\sigma$ is any fixed number greater than a.

Proof. Recall the definitions (10) and (11) of $K(x)$ and $H(x)$ respectively. The theorem will be proved by establishing the following string of equalities.

$$
\begin{aligned}
\left\langle\frac{1}{2 \pi i}\right. & \left.\int_{\sigma-i r}^{\sigma+i r} F(s) H(s t) d s, \phi(t)\right\rangle \\
\quad & \int_{0}^{\infty} \phi(t) d t \frac{1}{2 \pi} \int_{-r}^{r} F(s) H(s t) d \omega \quad(s=\sigma+i \omega) \\
& =\frac{1}{2 \pi} \int_{-r}^{r}\langle f(\tau), K(s \tau)\rangle \int_{0}^{\infty} \phi(t) H(s t) d t d \omega \\
& =\left\langle f(\tau), \frac{1}{2 \pi} \int_{-r}^{r} K(s \tau) \int_{0}^{\infty} \phi(t) H(s t) d t d \omega\right\rangle \\
& \rightarrow\langle f(\tau), \phi(\tau)\rangle .
\end{aligned}
$$

Since $\phi(t)$ is of compact support (30) is a repeated integral on $(t, \omega)$ and consequently (30) equals (31). Since by Theorem $1 F(s)$ is analytic, for fixed $r$ we can change the order of integration and arrive at (32). To which an application of Lemma 3 yields (33). Now, (33) goes into (34) by Lemma 4.

From the above inversion theorem the following uniqueness theorem can be deduced as a corollary.

Corollary. Let $F(s)=\mathscr{A}_{k, m} f$ for $\operatorname{Re} s>\sigma_{f}$, let $G(s)=\mathscr{M}_{k, m} g$ for $\operatorname{Re} s>\sigma_{g}$, and let $F(s)=G(s)$ for $\operatorname{Re} s>\max \left(\sigma_{f}, \sigma_{g}\right)$. Then in the sense of equality in $D^{\prime}(I), f=g$.

6. An operation-transform formula. Now, we shall obtain an operation-transform formula which may be used in solving certain 
integrodiffierential equations.

We define an operator $\Delta_{x}^{*}: \mathscr{J}_{a}^{k, m^{\prime}}(I) \rightarrow \mathscr{I}_{a}^{k, m^{\prime}}(I)$ by the relation

$$
\left\langle\Delta_{x}^{*} f(x), \phi(x)\right\rangle \triangleq\left\langle f(x), \Delta_{x} \phi(x)\right\rangle
$$

for all $f \in \mathscr{F}_{a}^{k, m^{\prime}}(I)$ and $\phi \in \mathscr{F}_{a}^{k, m}(I)$. Let us call $\Delta_{x}^{*}$ as the adjoint of the operator $A_{x}$ defined by (14). It can also be shown that for all $r=1,2,3, \cdots$ and $\phi(x) \in \mathscr{I}_{a}^{k, m}(I)$,

$$
\left\langle\left(\Delta_{x}^{*}\right)^{r} f(x), \phi(x)\right\rangle=\left\langle f(x),\left(\Delta_{x}\right)^{r} \dot{\phi}(x)\right\rangle .
$$

It can be readily seen from the definitions of the operators $\Delta_{x}$ and $\nabla_{x}$ given in $\S 2$ that if $f$ is a regular generalized function in $\mathscr{J}_{a}^{k, m^{\prime}}(I)$ generated by a member of $D(I)$, then

$$
\Delta_{x}^{*} f \equiv \nabla_{x} f .
$$

THEOREM 3. Let $F(s)$ be the distributional Meijer transform of $f$ for $\operatorname{Re} s>\sigma_{f}$, then for any positive integer $r$,

$$
\mathscr{C}_{k, m}\left[\left(\Delta_{x}^{*}\right)^{r} f\right]=(-s)^{r} F(s) .
$$

The proof of trivial.

7. Characterization of Meijer transforms. The following theorem gives a characterization of distributional Meijer transforms.

THEOREM 4 (Characterization). Let $\operatorname{Re} m \geqq 0$ and $\operatorname{Re} k \leqq$ $-\operatorname{Re} m<1 / 2$. Then a necessary and sufficient condition for a function $F(s)$ to be the Meijer transform of some generalized function according to our definition given in $\S 4$ is that there be a halfplane $\{s \mid \operatorname{Re} s \geqq b>0\}$ on which $F(s)$ is analytic and bounded according to

$$
|F(s)| \leqq P_{b}(|s|)
$$

where $P_{b}(|s|)$ is a polynomial in $|s|$ depending in general on the choice of $b$.

Proof. Necessity. By Theorem $1 F(s)$ is analytic function of $s$ for $\operatorname{Re} s>\sigma_{f}$. Choose two real numbers $a$ and $b$ such that $\sigma_{f}<$ $a<b$. Then, $K(s t) \in \mathscr{J}_{a}^{k, m}$ for $\operatorname{Re} s>b$. Now, by the boundedness property of generalized functions [14, pp. 18-19], there exist a constant $C$ and a nonnegative integer $r$ such that

$$
\begin{aligned}
|F(s)| & \leqq C \max _{0 \leqq n \leqq r} \rho_{n}(K(s t)) \\
& =C \max _{0 \leqq n \leqq r} \sup _{0<t<\infty}\left|e^{a t} t^{k+m} \Delta_{t}^{(n)}\left\{e^{-1 / 2 s t}(s t)^{-k-1 / 2} W_{k+1 / 2, m}(s t)\right\}\right|
\end{aligned}
$$




$$
\begin{aligned}
& =C \max _{0 \leqq n \leqq r} \sup _{0<t<\infty}\left|e^{a t} t^{k+m} s^{n} e^{-1 / 2 s t}(s t)^{-k-1 / 2} W_{k+1 / 2, m}(s t)\right| \\
& \leqq C|s|^{n-\lambda} r e^{\left|\lambda_{i}\right| \pi} \sup _{0<t<\infty}\left|e^{a t}(s t)^{m-1 / 2} e^{-1 / 2 s t} W_{k+1 / 2, m}(s t)\right|
\end{aligned}
$$

where $\lambda_{r}=\operatorname{Re}(m+k)$ and $\lambda_{i}=\operatorname{Im}(m+k)$. The inequality (36) now follows from Lemma 2.

Sufficiency. Let $q$ be a real number greater than 1 and let $n$ be a positive integer such that $n-q$ is greater than or equal to the degree of $P_{b}(|s|)$. Then, $s^{-n} F(s)$ satisfies the assumptions of Meijer's theorem stated in $\S 1$ and therefore, for $\operatorname{Re} s>c>b$,

$$
s^{-n} F(s)=\int_{0}^{\infty} g(t) e^{-1 / 2 s t} W_{k+1 / 2, m}(s t)(s t)^{-k-1 / 2} d t
$$

where

$$
g(t)=\frac{\Gamma(1-k+m)}{2 \pi i \Gamma(1+2 m)} \int_{c-i \infty}^{c+i \infty} s^{-n} F(s) e^{1 / 2 s t} M_{k-1 / 2, m}(s t)(s t)^{k-1 / 2} d s
$$

Now, consider the expression

$$
\begin{aligned}
\frac{g(t) e^{-c t}}{t^{k+m}\left(1+t^{-\lambda \cdot r}\right)}= & \frac{1}{2 \pi i} \frac{\Gamma(1-k+m)}{\Gamma(1+2 m)} \int_{c-i \infty}^{c+i \infty} s^{-n+k+m}\left(1+|s|^{-\lambda \cdot r}\right) F(s) \\
& \times\left[\frac{e^{-c t} e^{1 / 2 s t} M_{k-1 / 2, m}(s t)(s t)^{-m-1 / 2}}{\left(1+t^{-\lambda}\right)\left(1+|s|^{-\lambda_{r}}\right)}\right] d s .
\end{aligned}
$$

Using the series representation (1) and the asymptotic expansions (4) and (5) and following the technique of the proof of Lemma 2 it can be shown that

$$
\left|e^{-c t}(s t)^{-m-1 / 2} e^{1 / 2 s t} M_{k-1 / 2, m}(s t)\right| \leqq D\left(1+|s|^{-\lambda_{r}}\right)\left(1+t^{-\lambda_{r}}\right)
$$

on the line $s=c+i \omega,-\infty<\omega<\infty$ uniformly for all $t \in(0, \infty)$, where $D$ is a constant independent of $s$ and $t$. Furthermore,

$$
\begin{aligned}
\left|s^{-n-k+m}\left(1+|s|^{-\lambda_{r}}\right) F(s)\right| & \leqq\left(|s|^{-n} P_{b}(|s|)+|s|^{\lambda_{r}-n} P_{b}(|s|)\right) e^{\left|\lambda_{i}\right| \pi / 2} \\
& \leqq E\left(|s|^{-q}+|s|^{\lambda_{r}-q}\right),
\end{aligned}
$$

where $E$ is another constant. Since $q>1$ and $\lambda_{r} \leqq 0$, it follows that for any $d>c, e^{-d t} g(t)\left(1+t^{\lambda}\right)^{-1}$ is absolutely integrable on $0<t<\infty$, and consequently $e^{-d t} t^{-\lambda_{s}} g(t)$ is also absolutely integrable on the same interval. Hence $g(t)$ generates a regular distribution of $\mathscr{J}_{d}^{k, m^{\prime}}(I)$. Therefore, (37) represents a distributional Meijer transform for $\operatorname{Re} s>d$.

Now, let $f=\left(-\Delta_{x}^{*}\right)^{n} g$. Then, by Theorem 3,

$$
\mathscr{N}_{k, m}[f]=s^{n} \mathscr{N}_{k, m}[g]=F(s)
$$

for at least $\operatorname{Re} s>d$. This completes the proof. 
We conclude this section with the following structure theorem.

THEOREM 5. Let $f$ be an arbitrary element of $\mathscr{J}_{a}^{k, m}(I)$. Then there exist bounded measurable functions $g_{i}(x)$ defined for $x>0$ and for $i=0,1,2, \cdots, r$ where $r$ is some nonnegative integer depending upon $f$ such that for arbitrary $\phi \in D(I)$, we have

$$
\langle f, \phi\rangle=\left\langle-\sum_{i=0}^{r} \nabla_{x}^{i}\left[e^{a x} x^{k+m} D_{x}^{2} \int_{0}^{x} g_{i}(t) d t\right], \phi(x)\right\rangle,
$$

where $\nabla_{x}$ is the integrodifferential operator defined by (15).

Proof. The proof is analogous to a number of proofs available in the literature $[10$, pp. $272-274 ; 6, \mathrm{pp} .14-15]$ and therefore is omitted.

8. Applications. Now we will apply our inversion theory to the solution of certain integrodifferential equations.

(a) Solution of $P\left(\Delta_{x}^{*}\right) u=g$. Let $P$ be any polynomial. For $\operatorname{Re} m \geqq 0$ and $\operatorname{Re} k \leqq-\operatorname{Re} m<1 / 2$, consider the operational equation

$$
P\left(\Delta_{x}^{*}\right) u=g \quad 0<x<\infty
$$

where $g$ is a given Meijer transformable generalized function and $u$ is unknown generalized function.

Now to determine $u$, using (35) we apply the distributional Meijer transformation to (41) and get

$$
P(-s) U(s)=G(s)
$$

where $G(s)=\mathscr{C}_{k, m} g$ for $\operatorname{Re} s>\sigma_{g}$. Let $\sigma_{p}$ be the largest of the real parts of the roots of $P(-s)=0$. Then $G(s) / P(-s)$ satisfies hypotheses of Theorem 4 on some half-plane $\left\{s \mid \operatorname{Re} s \geqq b>\max \left(0, \sigma_{g}, \sigma_{p}\right)\right\}$ and hence it is a distributional Meijer transform of some $u \in \mathscr{I}_{b}^{k, m}$. We may apply the inversion formula (29) to get $u$. Thus

$$
\begin{aligned}
u(x)= & \lim _{r \rightarrow \infty} \frac{\Gamma(1-k+m)}{2 \pi i \Gamma(1+2 m)} \\
& \times \int_{\sigma-i r}^{\sigma+i r}[G(s) / P(-s)](s t)^{-k-1 / 2} e^{1 / 2 s t} M_{k-1 / 2, m}(s t) d s
\end{aligned}
$$

in the sense of equality in $D^{\prime}(I)$, which is a solution of (41). This solution is in fact a restriction of $u \in \mathscr{J}_{b}^{k, m^{\prime}}(I)$ to $D(I)$, and is unique in view of the corollary following Theorem 2.

By arguments preceding Theorem 3 one can easily verify that $u$ as determined by (42) is also a solution to the distributional 
integrodifferential equation

$$
P\left(\nabla_{x}^{k, m}\right) u=g .
$$

(b) Solution of $P\left(\nabla_{x}^{-k, m}\right) \dot{\phi}=\psi$. Suppose that $\psi$ is a given Meijer transformable conventional function possessing the asymptotic properties:

$$
\begin{aligned}
\psi(x) & =O\left(e^{a x}\right) \quad x \longrightarrow \infty \\
& =O\left(x^{\rho}\right) \quad x \longrightarrow 0+
\end{aligned}
$$

where $a>0$ and $\operatorname{Re}( \pm m-k)+\rho+1>0$. We wish to find $\phi$ such that

$$
P\left(\Delta_{x}^{-k, m}\right) \phi=\psi
$$

If we assume that

$$
\begin{aligned}
\dot{\phi}^{(r)}(x) & =O\left(e^{b x}\right), & & x \longrightarrow \infty \\
& =O\left(x^{\beta-r}\right), & & x \longrightarrow 0+
\end{aligned}
$$

for each $r=0,1,2, \cdots$, we can apply Meijer transform (8) to (44) and get

$$
\int_{0}^{\infty} P\left(\Delta_{x}^{-k, m}\right) \dot{\phi}(x) K(s x) d x=\Psi(s)
$$

where $\operatorname{Re} s>\max (a, b)$ and $\Psi(s)$ is a Meijer transform of $\psi(x)$. Now, using the formula [2, p. 733]

$$
\frac{d}{d z}\left\{z^{k} e^{-1 / 2 z} W_{k, m}(z)\right\}=-z^{k-1} e^{-1 / 2 z} W_{k+1, m}(z)
$$

and integrating by parts the left-hand side of (45), we get

$$
P(-s) \Phi(s)=\Psi(s)
$$

where $\Phi(s)$ is the Meijer transform of $\phi(x)$. If we further assume that $\operatorname{Re} s \geqq c>\max \left(a, b, \sigma_{q}\right)$, where $\sigma_{q}$ is the largest of the real parts of roots of $P(-s)=0$, we find that $\Psi(s) / P(-s)$ satisfies conditions of Meijer's theorem (given in $\S 1$ ), and hence is the Meijer transform of some function $\phi(x)$ defined by

$$
\begin{aligned}
\phi(x)= & \frac{\Gamma(1-k+m)}{2 \pi i \Gamma(1+2 m)} \int_{c-i \infty}^{c+i \infty} e^{1 / 2 x s} M_{k-1 / 2, m}(x s)(x s)^{-k-1 / 2} \\
& \times[\Psi(s) / P(-s)] d s .
\end{aligned}
$$

Following the technique of proof of sufficiency part of Theorem 4 it can be shown that $\phi(x)$, as a regular distribution, is a member of $\mathscr{J}_{d}^{k, m^{\prime}}(I)$, where $d>c$. 
ACKNOWLEDGMENT. The author expresses his gratefulness to the referee for his constructive criticisms. This work is partially supported by U.G.C. (New Delhi) grant No. 8711.

\section{REFERENCES}

1. A. Erdélyi, (Editor). Tables of Integral Transforms vol. II, McGraw-Hill Book Co., New York (1954), pp. 431-432.

2. C. S. Meijer, Eine nene erweiterung der Laplace transformation, Nederl. Akad. Wetench. Proc. Series A, 44 (1941), 727-739.

3. J. N. Pandey, On the Stieltjes transform of generalized functions, Proc. Camb. Phil. Soc., 71 (1972), 85-96.

4. —, An extension of Haimo's form of Hankel convolutions, Pacific J. Math., 28 (1969), 641-651.

5. R. N. Pandey, A generalization of Meijer transform, Proc. Camb. Phil. Soc., 67 (1970), 339-345.

6. R. S. Pathak, Transformée de Varma des fonctions généralisées, Bull. Sci. Math., $2^{e}$ série 99 (1975), 3-16.

7. L. J. Slater, Confluent hypergeometric functions, Cambridge University Press (1960).

8. H. M. Srivastava, A relation between Meijer and generalized Hankel transforms, Math. Japan, 11 (1966), 11-13.

9. H. M. Srivastava and O. D. Vyas, A Theorem relating generalized Hankel and Whittaker transforms, Nederl. Akad. Wetensch. Proc. Ser. A, 72 No. 2 (1969), 140-144. 10. F. Treves, Topological Vector Spaces, Distributions and Kernels, Academic Press, New York (1967).

11. R. S. Varma, On a generalization of Laplace integral, Proc. Nat. Acad. Sc., India. Sec. A, 20 (1951), 209-216.

12. E. T. Whittaker and G. N. Watson, A course of modern analysis, Cambridge University Press, Fourth Edn. (1962).

13. A. H. Zemanian, $A$ distributional $K$ transformation, SIAM J. Appl. Math., 14, No. 6 (1966), 1350-1365.

14. - Generalized Integral Transformations, Interscience Publishers, New York (1968).

Received April 22, 1976 and in revised form April 21, 1978.

BANARAS HINDU UNIVERSITY

VARANASI 221005, INDIA 


\section{PACIFIC JOURNAL OF MATHEMATICS}

\section{EDITORS}

RICHARD ARENS (Managing Editor)

University of California

Los Angeles, CA 90024

Charles W. Curtis

University of Oregon

Eugene, OR 97403

C. C. MOORE

University of California

Berkeley, CA 94720
J. DUGUNDJI

Department of Mathematics

University of Southern California

Los Angeles, CA 90007

R. FINN and J. MILGRAM

Stanford University

Stanford, CA 94305

\section{ASSOCIATE EDITORS}

\section{E. F. BeCKENBACH}

B. H. NeumanN

F. WOLF

K. YoSHIDA

\section{SUPPORTING INSTITUTIONS}

\author{
UNIVERSITY OF SOUTHERN CALIFORNIA \\ STANFORD UNIVERSITY \\ UNIVERSITY OF HAWAII \\ UNIVERSITY OF TOKYO \\ UNIVERSITY OF UTAH \\ WASHINGTON STATE UNIVERSITY \\ UNIVERSITY OF WASHINGTON
}

The Supporting Institutions listed above contribute to the cost of publication of this Journal, but they are not owners or publishers and have no responsibility for its content or policies.

Mathematical papers intended for publication in the Pacific Journal of Mathematics should be in typed form or offset-reproduced, (not dittoed), double spaced with large margins. Please do not use built up fractions in the text of the manuscript. However, you may use them in the displayed equations. Underline Greek letters in red, German in green, and script in blue. The first paragraph or two must be capable of being used separately as a synopsis of the entire paper. Items of the bibliography should not be cited there unless absolutely necessary, in which case they must be identified by author and journal, rather than by item number. Manuscripts, in triplicate, may be sent to any one of the editors. Please classify according to the scheme of Math. Reviews, Index to Vol. 39. All other communications should be addressed to the managing editor, or Elaine Barth, University of California, Los Angeles, California, 90024.

50 reprints to each author are provided free for each article, only if page charges have been substantially paid. Additional copies may be obtained at cost in multiples of 50 .

The Pacific Journal of Mathematics is issued monthly as of January 1966. Regular subscription rate: $\$ 72.00$ a year (6 Vols., 12 issues). Special rate: $\$ 36.00$ a year to individual members of supporting institutions.

Subscriptions, orders for numbers issued in the last three calendar years, and changes of address should be sent to Pacific Journal of Mathematics, P.O. Box 969, Carmel Valley, CA 93924, U.S.A. Older back numbers obtainable from Kraus Periodicals Co., Route 100, Millwood, NY 10546.

PUBLISHED BY PACIFIC JOURNAL OF MATHEMATICS, A NON-PROFIT CORPORATION

Printed at Kokusai Bunken Insatsusha (International Academic Printing Co., Ltd.). 8-8, 3-chome, Takadanobaba, Shinjuku-ku, Tokyo 160, Japan. 


\section{Pacific Journal of Mathematics}

\section{Vol. 80, No. $2 \quad$ October, 1979}

K. Adachi, On the multiplicative Cousin problems for $N^{p}(D) \ldots \ldots \ldots \ldots 297$

Howard Banilower, Isomorphisms and simultaneous extensions in $C(S) \ldots 305$

B. R. Bhonsle and R. A. Prabhu, An inversion formula for a distributional

finite-Hankel-Laplace transformation ................... 313

Douglas S. Bridges, Connectivity properties of metric spaces.......... 325

John Patton Burgess, A selection theorem for group actions ........... 333

Carl Claudius Cowen, Commutants and the operator equations

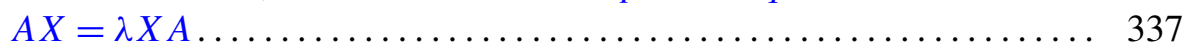

Thomas Curtis Craven, Characterizing reduced Witt rings. II .......... 341

J. Csima, Embedding partial idempotent d-ary quasigroups ............ 351

Sheldon Davis, A cushioning-type weak covering property ............ 359

Micheal Neal Dyer, Nonminimal roots in homotopy trees ............. 371

John Erik Fornaess, Plurisubharmonic defining functions ........... 381

John Fuelberth and James J. Kuzmanovich, On the structure of finitely

generated splitting rings .......................... 389

Irving Leonard Glicksberg, Boundary continuity of some holomorphic

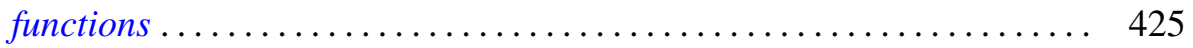

Frank Harary and Robert William Robinson, Generalized Ramsey theory.

IX. Isomorphic factorizations. IV. Isomorphic Ramsey numbers .......

Frank Harary and Allen John Carl Schwenk, The spectral approach to determining the number of walks in a graph...........

David Kent Harrison, Double coset and orbit spaces ..... . .

Shiro Ishikawa, Common fixed points and iteration of commuting

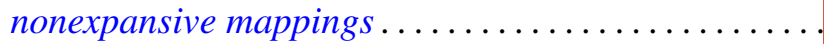

Philip G. Laird, On characterizations of exponential polynomials ........ 503

Y. C. Lee, A Witt's theorem for unimodular lattices ...........

Teck Cheong Lim, On common fixed point sets of commutative

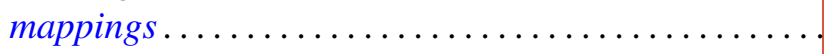

R. S. Pathak, On the Meijer transform of generalized functions ...

T. S. Ravisankar and U. S. Shukla, Structure of $\Gamma$-rings . . .

Olaf von Grudzinski, Examples of solvable and nonsolvable convolution

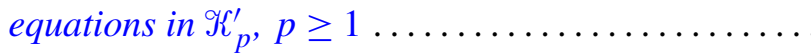

